\title{
Kinematical Analysis on the two-handed backhand stroke skills of excellent Tennis Player Ramos
}

\author{
Xiaozhen Zhang ${ }^{1}$, Jihe Zhou $^{2, *}$ \\ Chengdu, Chengdu sport institute postgraduate school, 610041 \\ E-mail:1619186592@qq.com \\ ${ }^{*}, 2$ Corresponding author: jihezhou123@163.com
}

Key words: Ramos; tennis; two-handed backhand; kinematics analysis

Abstract:This paper carried out kinematical analysis for the two-handed backhandmovement of the 2016 ATP Chengdu Tennis Open final men's players Ramos byusing three-dimensional camera analysis, to explore the characteristics of tennis two-handed backhand, hoping to provide the majority of tennis Coaches, athletes and enthusiasts with some references and improve the skill of two-handed backhand. In order to reduce inconveniencewhile analyzing, the movementwill be divided into four moments and three stages. The paper analyze the movement features specifically from thebackswing, swing and follow through these three stages. The results show that Ramos 'smovement is reasonable.The body' s center of gravity descends obviously, and the backswing reveals the mechanics principle that the large joints leads to the small joints. The whipping movement is obvious during the swinging stage, but the body' stwist is smaller. During the follow through stageRamos' stop movement is obvious, but at the end of the moment the legs are still in a state of bending, especially the right knee is still in a lower position, which shows that Ramos didn't stretch hip and knees sufficientlyduring the follow through stage.

\section{Introduction}

Two-handed backhand is one of the most basic and most important tennis skills, as well as the base line fore hand, which can play difficult and high-quality forcing shot. There is few analysis of two-handed backhand movement through the three-dimensional analysis software. Therefore, it is particularly important to carry out kinematic analysis of the movement of the two-handed backhand of excellent tennis player in the game.

\section{Objects and methods}

\section{Study object}

This paper takes 2016 ATP Chengdu Tennis Open final male player Ramos as the object of study. His basic information is as follows: Spanish, his height is $1.88 \mathrm{~m}$, weight is $80 \mathrm{~kg}$, awarded the runner-up trophy in the tournament.

\section{Study methods}

In 2016 Chengdu Tennis Open final scene,we used two JVC GC-PX10AC cameras at 50 frames / sec speed shooting the two-handed backhand process of Ramos. It's three-dimensional fixed-point shooting. No. 1 machine was in the back of side line. No. 2 machine was in the front of 
the side line. The main optical axis angle between the two cameras is about $65^{\circ}$. We adopted 3-D Signal TEC V3.2HDC analysis system to analyze thevideo while using the European Dempster Human body as model (16 links, 21 joints). According to the research needs, the analysis added two measurement pointsof the top of the racket and the ball. We used series analysis. Thedata was smoothly processed by low-pass filter methods. The cut-off frequency is $8 \mathrm{~Hz}$.

\section{Results and Discussions}

Ramos adopted a semi-closed footwork. Left hand is the advanced hand (left hand is the hitting hands).The movements from the body sideways to prepare to the end of the swing are smooth and stretched. But there are still some problems.

The stage from the end of the preparation to the end of the backswing is called backswing stage.

In this stage, the left hip angle was $84.1^{\circ}$, the right hip angle is $131.7^{\circ}$, the left knee angle is $136.1^{\circ}$, the right knee angle is $112.2^{\circ}$, and theheight of the center of gravityis $0.783 \mathrm{~m}$. The data demonstrated that Ramos's hip and knee joint are in a buckling state with weight significantly downward when he finished backswing. Meanwhile, Ramos' right knee angle is $112.2^{\circ}$, left knee angle is $136.1^{\circ}$,hind leg joint angle is smaller than the front leg joint angle, indicating Ramos' weight backward when he finished backswing, which is conducive to push off with his back leg. It's conducive to store more elastic potential energy to obtain greater angular momentum.

Left and right shoulder and elbow joint angle can reflect the distanceof racket and the bodywhen backswing is finished. A reasonable range of backswing is very important for swing. In the caseof same extension, the elbow angle is inversely proportional to shoulder angle. Shoulder angle is small while elbow angle is large, elbow angle is small while shoulder angle is big. This is the key to fully backswing. In this stage, the left shoulder angle of Ramos is $64.4^{\circ}$, the right shoulder angle is $34.1^{\circ}$, the left elbow angle is $174.1^{\circ}$, and the right elbow angle is $132.1^{\circ}$. Therefore, Ramos' elbow is larger than the shoulder joint which conformswithbig shoulder angle and small elbow angle principle in the case of same extension. The literaturerevealed that Djokovic's left shoulder angle is $111.2^{\circ}$, the right shoulder angle is $37.9^{\circ}$, the left elbow angle is $169.8^{\circ}$, and the right elbow angle is $107.5^{\circ}$ when he finished backswing. The data shows that the two athletes' arms are in the stretchedstate. The racket has been away from the body. The surmount degree is enough to increase thedistance of swing, which is conducive to get a larger line speed when swing forward.

In the stage of backswing, the joint speed increased in sequence when backswing is fast. The literature revealed that Djokovic's left and right shoulder speed are $1.32 \mathrm{~m} / \mathrm{s}$ and $1.36 \mathrm{~m} / \mathrm{s}$, left and right elbow speed are $1.48 \mathrm{~m} / \mathrm{s}$ and $1.88 \mathrm{~m} / \mathrm{s}$, left and right wrist speed are $1.94 \mathrm{~m} / \mathrm{s}$ and $1.75 \mathrm{~m} / \mathrm{S}$, head speed is $4.44 \mathrm{~m} / \mathrm{s}$. The speed of each joint is also increasing in turn. The results declared that 
the two players' movement meet the mechanics ofthe large joints driving small joints during backswing stage, which will help produce faster beat speed when the racket contact with ball.

The stage from the end of the backswing to the contact moment is called swing.

During the swing stage, Ramos'sleft hip angle is $114.4^{\circ}$, right hip angle is $157.4^{\circ}$, left knee angle is $126.0^{\circ}$, right knee angle is $96.1^{\circ}$, the height of center of gravity is $0.807 \mathrm{~m}$, which has risen compared with that when he finished backswing. It means that the height of center of gravity of Ramos is rising forward and upward when he hit the ball. The literature shows that when the ball hits the racket Djokovic's left and right hip angle are $175.9^{\circ}$ and $158.7^{\circ}$, left and right knee angle are $146.7^{\circ}$ and $174.3^{\circ}$.At this moment Djokovic's hip and knee joint angle is larger. He is almost standing when hitting the ball. Compared with Ramos' hip and knee angle when the ball hits the racket, Ramos has a lower center of gravity, which is more conducive to lower limbspedal pull force and get greater angular momentum.

When the backswing stage finished, Ramos' left shoulder angle is $64.4^{\circ}$. When the ball hits the racket, the left shoulder angle is $25.2^{\circ}$, whichgradually reducedin the swing stage and reached the minimum when the rackethits the ball. At the end of the backswing, the right shoulder angle is $34.1^{\circ}$. When the ball hits the racket the right shoulder angle is $40.3^{\circ}$, and it is gradually increased in the swinging stage, reaching the maximum at the time of the ball hits the racket. The above data shows that when he pushes off and switch the body to hit the ball, Ramos achieved the purpose of raising the shoulders and elbows. The movement is relatively smooth.

The speed of the joints on the right side of the body, the right elbow, the right wrist and the top of racketare $2.73 \mathrm{~m} / \mathrm{s}, 5.04 \mathrm{~m} / \mathrm{s}, 8.81 \mathrm{~m} / \mathrm{s}$ and $20.73 \mathrm{~m} / \mathrm{s}$. It can be seen that Ramos's speed of the body are gradually increasing when the ball hits the racket. The speed of the top of racket reach the maximum of $20.73 \mathrm{~m} / \mathrm{s}$. It means that at the end of the stage,backswing to the ball hits the racket, the whipping movement is obvious which empowers the hitting, and bolster the attacking power.

The twistmagnitude of the body affects hitting power. During swing stage, athletes should try to reverse the upper body. The greater the shoulderand hipangle are, the greater momentum they will get. At this stage Ramos' body rotating range is $27.5^{\circ}$ while Djokovic's is $54.1^{\circ}$. Ramos's range is relatively smaller.

From the timing of the ball hits the racket to the end of the swing is the follow through phrase.

Ramos' left hip angle is $176.2^{\circ}$ when the follow through phrase ends, the right hip angle is $157.9^{\circ}$. Compared with the left and right hip angles $111.4^{\circ}$ and $157.4^{\circ}$. When the ball hits the racket they increased. The left knee angle is $146.8^{\circ}$, right knee angle is $109.4^{\circ}$, and compared with knee angle $126.0^{\circ}, 96.1^{\circ}$ when the ball hits the racket significantly increased. In the follow through stage, hip and knee joint angle increased, the height of the bodyincreasedfrom $0.807 \mathrm{~m}$ to $0.948 \mathrm{~m}$.It shows that the center of gravity driven by ball continues to pass the power up after Romos hit the 
ball, but at the end of the moment Ramos' left and right leg is still in a state of bending, especially the right knee is still in a lower position. It means that in the follow through stage Ramos didn't stretch hip and knee sufficiently, which is not conducive to deliver the momentum completely.

\section{Conclusions}

The above analysis of the results showsthat:during the backswing stage Ramos' movements are more reasonable. When he finished backswing, the body center of gravity decreased backward significantly, which is conducive for hind legs to hit the ground and store more elastic potential energy in order to obtain larger angular momentum.

Swing stage: when the ball hits the racket Ramos's center of gravity is $0.807 \mathrm{~m}$, which compared with the center gravity height of $0.783 \mathrm{~m}$ in former stage has increased. It means that the center of gravity weight of Ramos is rising forward and upward when he hit the ball. It can be seen that Ramos' speed of the body are gradually increasing when the ball hits the racket. The speed of the top of racket reaches the maximum of $20.73 \mathrm{~m} / \mathrm{s}$. It means at the stage of end of the backswing to the ball hits the racket, the whipping movement is obvious. But Ramos at this stage moves to a lesser extent.

Follow through stage: Ramos' stop movement is obvious. But at the end of the follow through Ramos' left and right leg is still in a state of bending, especially the right knee is still in a low position. It means that in the follow through stage Ramos didn't stretch hip and knees sufficiently, which is not conducive to deliver the momentum obtained completely.

In general, when Ramos uses two-handed backhand skills, the overall movement is stretched and smooth, which can be a good example for the majority of tennis coaches, athletes, enthusiasts to learn from. However, in the follow through stage Ramos didn't stretch hip and knees sufficiently and in the swing stage, Ramos's body didn't twist to a considerable extent which needs to pay attention to and avoid the same mistakes.

\section{References}

[1] Wang Wei. Kinematical Analysis of Two-handed Backhand Stroke skills of Outstanding Tennis Players in Part of China[D].Shenyang Sport University.2012

[2] Lin Feng. Motion Analysis of Two-handed Backhand Batting of Top Spin in Tennis[J].Journal of Xi'an Sport University.2010, 8 (5):77-78.

[3]Zhixiang Tao. Tennis tutorial[M].Beijing: Beijing Sport University

Press,2006:138.

[4]Xiaofei Liu. On How to Improve the Two-handed Backhand Stroke Skills[J].Journal of Jinzhou Normal University, 2002,23(1).

[5]Kaijun Wang. Research and Technical Analysis on Training of Three Kinds of Latest 
Two-handed Backhand Tennis skills[ J].Journal of Wuhan Sport University,2006 40 ( 9): 71- 73.

[6]GuoJie. Discussion on Teaching Method of Anti - shot and Shot Technique in

Tennis[J].Contemporary Sports Science and Technology,2014(6):42-43,46.

[7]Pan Shen, Hongcheng Zhang. Comparative Analysis of Technical Characteristics and

Biomechanical Characteristics of Two Tennis Bottom Line Hitting. 